\title{
Physical Examination for Men and Women with Urological Chronic Pelvic Pain Syndromes: A MAPP Network Study
}

\author{
Claire C. Yang ${ }^{a}$, Jane L. Miller ${ }^{a}$, Adam Omidpanah ${ }^{b}$, and John N. Krieger \\ aDepartment of Urology, University of Washington, Seattle, WA USA \\ bInitiative for Research and Education to Advance Community Health (IREACH), Washington \\ State University, Seattle, WA USA
}

\begin{abstract}
Objectives-To examine the feasibility of implementing a standardized, clinically relevant genitourinary examination for both men and women and to identify physical examination findings characteristic of urological chronic pelvic pain syndromes (UCPPS).

Methods-This study analyzed two samples: men and women with UCPPS who participated in the MAPP Research Network Epidemiology and Phenotyping (EP) Study, and age-matched controls who were either positive for chronic fatigue syndrome or healthy (pain-free). We compared physical examination findings in both positive and healthy controls to UCPPS cases, findings from both the EP examinations and from an extended genitourinary examination.
\end{abstract}

Results-EP and extended examinations were performed on 143 participants: 62 UCPPS cases (30 women, 32 men), 42 positive controls (15 women, 27 men), and 39 healthy controls (22 women, $17 \mathrm{men})$. EP examinations showed that pelvic floor tenderness was more prevalent in cases $(55.0 \%)$ than in positive (14.6\%) or healthy controls (10.5\%). Extended examinations revealed specific areas of tenderness in the pelvic floor musculature. Cases were also more likely than healthy controls to report tenderness in multiple areas, including suprapubic, symphysis pubis, and posterior superior iliac spine, and on bimanual examination. No comparative findings were specific to biological sex, and no evidence of pudendal neuropathy was observed on extended examination of cases or controls.

Conclusions-The extended genitourinary examination is an easily administered addition to the assessment of men and women during evaluation for UCPPS. Physical findings may help to better categorize UCPPS patients into clinically relevant subgroups for optimal treatment.

Corresponding Author: Claire C. Yang, MD, Department of Urology, Box 356510, University of Washington, Seattle, WA 98195-6510 USA, phone: 1-206-598-2827, fax: 1-206-744-4709, cyang@u.washington.edu.

Publisher's Disclaimer: This is a PDF file of an unedited manuscript that has been accepted for publication. As a service to our customers we are providing this early version of the manuscript. The manuscript will undergo copyediting, typesetting, and review of the resulting proof before it is published in its final citable form. Please note that during the production process errors may be discovered which could affect the content, and all legal disclaimers that apply to the journal pertain.

The authors all declare no other financial disclosures. 


\section{Keywords}

physical examination; interstitial cystitis; bladder pain syndrome; chronic prostatitis; male chronic pelvic pain syndrome

\section{Introduction}

The Multidisciplinary Approach to the Study of Chronic Pelvic Pain (MAPP) Research Network was established to provide new perspectives on the clinical features and pathogenesis of urological chronic pelvic pain syndrome (UCPPS). ${ }^{1}$ This term was coined by the MAPP Research Network to encompass conditions commonly known as interstitial cystitis/bladder pain syndrome (IC/BPS) and chronic prostatitis/chronic pelvic pain syndrome (CP/CPPS). In contrast to traditional nomenclature, UCPPS acknowledges that these syndromes, which are characterized by pain and voiding symptoms, occur in both sexes and are not specific to the bladder or prostate. ${ }^{2}$ Moreover, IC/BPS and CP/CPPS have more clinical overlap than was formerly thought. MAPP Network researchers have found that a patient's sex is far less important than other factors in determining clinical presentation. ${ }^{3}$

The traditional concept of IC/BPS and CP/CPPS emphasized evaluation of pain sources distinct to each sex. Thus, many providers think in terms of separate genitourinary exams for men and women. The newer concept of UCPPS is supported by evidence that men and women with UCPPS appear to share certain physical examination findings. Men with CP/ CPPS are more likely to have abnormal pelvic findings than pain-free men, including muscle spasms, increased muscle tone and tension, and tenderness with palpation of the pelvic muscles. ${ }^{4-6}$ Women with IC/BPS have a high prevalence of pelvic floor myofascial tenderness, and these findings correlate with scores for pelvic floor dysfunction. ${ }^{7,8}$ Treatment of pelvic floor myalgia with physical therapy has improved outcomes for IC/BPS, ${ }^{9}$ while chemo-denervation of pelvic floor muscles has mitigated symptoms of CP/CPPS. ${ }^{10}$ Thus, manifestations of UCPPS in the pelvic floor and regions beyond can be identified on physical examination, and these findings might guide therapy.

Part of the data collected by the MAPP Network included brief, sex-specific examinations for men and women. On the basis of newer concepts of UCPPS, our MAPP Network study site conducted an extended genitourinary examination for both men and women in addition to the sex-specific examinations. Our objectives were to 1) assess the feasibility of an extended genitourinary examination for men and women undergoing evaluation for UCPPS, and 2) identify physical findings characteristic of UCPPS.

\section{Materials and Methods}

This single-site, cross-sectional investigation was conducted as an ancillary project of the MAPP Research Network Epidemiology and Phenotyping Study (MAPP EP Study), a multisite longitudinal observation study. Details of the MAPP EP study, including enrollment criteria, are provided elsewhere. ${ }^{11}$ All study procedures were approved by our medical center's Institutional Review Board, and all participants provided written informed consent. 
Study participants were prospectively enrolled as part of the larger MAPP EP Study. Cases were men and women with UCPPS; controls were drawn from two age- and sex-matched samples. "Positive controls" were patients diagnosed with chronic fatigue syndrome, a condition that includes chronic pain as a prominent symptom, while "healthy controls" had no prior diagnosis of any chronic pain condition. As part of the MAPP EP study, we performed separate examinations for men and women ("EP examinations"), as determined by the MAPP Steering Committee, plus an extended, standardized genitourinary examination for both sexes.

The EP examinations included measurement of height, weight, and blood pressure. Abdominal, external genital, and rectal or bimanual examinations were performed and findings designated as normal or abnormal, with the determinations left to the examiner's discretion. Tenderness in muscles of the pelvic floor (yes/no) was evaluated without regard to specific regions or muscle groups. In women, findings were recorded for the presence of a uterus and the degree of pelvic organ prolapse (above or below the hymenal ring). In men, findings were recorded for suprapubic tenderness (yes/no), penis (circumcised or not), prostate (enlarged, irregular, or tender) and scrotum (varicocele, hydrocele, mass, or hernia). 11

Our extended examination was designed for both men and women to supplement the EP examinations. It comprised assessment of 1) pelvic tenderness, specifically: in the perineal body, levator, obturator, and urogenital diaphragm muscles, in the pelvic organs via bimanual examination, and at specified bony pelvis points, 2) extra-pelvic, regional tenderness, including abdomen, flank, and back; 3) sensory function of the pudendal nerve, and 4) motor function of the pudendal nerve/pelvic floor. (For list of examination points, see Table 2.)

For both EP and extended examinations, tenderness was defined as a painful response to manual palpation. The examiner applied finger pressure to each examination point to produce discernible movement of muscle or organ tissue. No attempt was made to standardize the depth of palpation or the degree of pressure. If a participant reported pain on palpation when questioned, tenderness was recorded as present in that area.

Pelvic muscle tenderness was tested in both men and women at the perineal body, midway between the anus and the inferior edge of the scrotum in men, and the inferior edge of the vaginal introitus in women. In men, the pelvic floor muscles were palpated through the rectum: the urogenital diaphragm muscles were palpated anteriorly at the prostate apex; the obturator muscles were palpated anterior and laterally; and the levator muscles were palpated posteriorly (Figure 1). In women, these muscles were palpated through the vagina: the urogenital diaphragm muscles were palpated lateral to the urethra, approximately $2 \mathrm{~cm}$ proximal to the meatus on the anterior vaginal wall; the obturator muscles were palpated laterally, and the levator muscles were palpated posteriorly, toward the lateral aspects of the rectum (Figure 2).

The pudendal nerve is the primary mediator of somatosensory and skeletal motor function in the pelvis. Pudendal sensory function was measured by examining genital sensation (labia 
majora/penis and scrotum) in response to 1) a light touch using a standard cotton-tipped applicator and 2) a prick by a standard safety pin (both graded as present, absent, or equivocal), as well as by testing the bulbocavernosus reflex and anal wink (graded as present, absent, or equivocal). Pelvic motor function was assessed by voluntary pelvic floor contraction (graded as good, weak, or absent). Pudendal sensory and motor function were tested bilaterally. Currently, no standard methods are available to evaluate pelvic visceral sensory or motor function on physical examination.

Examinations were conducted by six physicians. All were trained to perform both the EP and the extended examinations in a standard manner, and were assigned study participant examinations based on schedule availability. Because these physicians conducted histories and physical examinations for each participant, they could not be blinded to individual status.

Data on demographics and other characteristics were collected, including age, sex, selfreported race and ethnicity, employment, marital status, symptom duration, body mass index, and blood pressure. Physical findings were presented by using counts and percentages. Percentages were based on the number of participants with data for the particular physical finding. For each examination element, Pearson chi-square tests for differences in percentages were performed to compare cases separately to positive controls and to healthy controls. Statistical significance was declared with a 5\% type 1 error rate. No correction was made for multiple comparisons. Analyses were done in $\mathrm{R}$ version 3.2.5 ( $\mathrm{R}$ Foundation for Statistical Computing, Vienna, Austria). ${ }^{12}$

\section{Results}

We examined 143 participants: 62 UCPPS cases ( 30 women, 32 men), 42 positive controls (15 women, $27 \mathrm{men}$ ), and 39 healthy controls ( 22 women, $17 \mathrm{men}$ ) (Table 1). Cases were more likely to be married or with a partner $(64.5 \%)$ than positive or healthy controls $(35.7 \%$ and $38.5 \%$, respectively). However, positive controls were more likely to be unemployed (26.2\%) than cases $(9.7 \%)$ or healthy controls $(15.4 \%)$, more likely to be disabled (31\%) than cases $(11.3 \%)$ or healthy controls $(0 \%)$, and more likely to be obese $(28.6 \%)$ than cases $(18 \%)$ or healthy controls $(15.8 \%)$.

All participants underwent the EP examinations and the extended genitourinary examination. None of the physicians reported difficulty conducting the examinations, and participants were allowed to omit any portion of either physical examination. The extended examination took 3-4 minutes to perform. Few values were missing for any examination point, except for the bimanual examination to determine pelvic organ tenderness, as part of the extended examination. Healthy male controls were not asked to undergo the bimanual examination, and results were recorded in only three male cases and two male positive controls. Female participants underwent the bimanual examination, but many female positive and healthy controls did not have data for the bimanual tenderness determination. 


\section{EP Examination Findings (Table 2, upper)}

Cases had more pelvic muscle tenderness (55\%) than positive or healthy controls (14.6\% and $10.5 \%$ respectively, $\mathrm{p}<0.05$ ). Cases were also more likely to have an abnormal abdominal examination than healthy controls, although the definition of "abnormal" was not specified in the MAPP EP Study protocol. The EP examinations returned no other differences between cases and controls.

\section{Extended Examination Findings (Table 2, lower)}

Compared to healthy controls, cases were statistically more likely to report tenderness in the suprapubic region, symphysis pubis, and posterior superior iliac spine, as well as on bimanual examination. As in the EP examinations, cases were more likely to report pelvic floor tenderness than positive or healthy controls in the extended examination. But the extended examination identified tenderness in specific regions, and in each one, prevalence was statistically significantly higher in cases. In the levator muscles, the prevalence of tenderness was $45.6 \%$ in cases, $12.2 \%$ in positive controls, and $5.4 \%$ in healthy controls; in the obturator muscles, prevalence was $40.4 \%$ in cases, $9.8 \%$ in positive controls, and absent in healthy controls; and in the urogenital diaphragm, prevalence was $40.4 \%$ in cases, $12.2 \%$ in positive controls; and absent in healthy controls.

Most participants (>95\% in each group) had intact pudendal nerve sensory and motor function. The occasional abnormal finding, for example, an absent bulbocavernosus reflex, were not neuropathic in the context of other results. In contrast to the reported tenderness described above, we found no difference between cases and either group of controls in the sensory or motor function of the pudendal nerve.

To examine potential gender differences, we analyzed men and women separately. The EP examination found more pelvic floor tenderness in male and female cases than in either group of controls (Supplemental Table 1). Among male cases, $47 \%$ had pelvic floor tenderness, compared to $8 \%$ of positive controls and $6 \%$ of healthy controls. Among female cases, $63 \%$ had pelvic floor tenderness, compared to $27 \%$ of positive controls and $14 \%$ of healthy controls. Among male cases, $22 \%$ had prostatic tenderness (all diagnosed with $\mathrm{CP} /$ CPPS), compared to none of the positive or healthy controls (not statistically significant). The extended examination detected higher rates of tenderness in all three major pelvic floor muscle groups (levator, obturator, urogenital diaphragm) in male and female cases than in healthy controls ( $\mathrm{p}<0.5$ for all comparisons). Men declined bimanual tenderness examinations at high rates, and very few participants reported perineal body tenderness, suggesting that these assessments might not prove useful during a UCPPS clinical examination.

\section{Comment}

Our extended genitourinary examination proved feasible for men and women evaluated for UCPPS. By administering both the EP examinations and the extended examination, we were better able to examine and describe tenderness in multiple anatomic regions. Our combination of both examinations proved clinically relevant, as it enabled us to rule out 
identifiable causes of pelvic pain and to assess both regional and pelvic tenderness. The extended examination also proved straightforward, even for providers who were not urogenital specialists, and did not require identification of explicit anatomic points of tenderness. Our approach is quite distinct from a tender point examination as defined by the American College of Rheumatology. ${ }^{13}$ The extended examination does not require any special determinants of tenderness (e.g., scales of tenderness, algometers), only notation of the presence or absence of patient-reported tenderness.

We were also able to identify physical findings characteristic of UCPPS. The most prominent was pelvic floor tenderness. During the EP examination, pelvic floor tenderness was reported by $55 \%$ of cases, $14.4 \%$ of positive controls, and $10.5 \%$ of healthy controls, with a high rate of prevalence in cases in both men and women. Pelvic floor tenderness in females with UCPPS may not be a new concept for many providers, but generally clinicians do not appreciate the high prevalence of pelvic floor tenderness in males with UCPPS. Pelvic bony tenderness and extra-pelvic tenderness were also more prevalent in cases than in healthy controls. Notably, the presence or absence of pelvic floor tenderness and other local and regional areas of tenderness are criteria currently under evaluation for distinguishing subsets of UCPPS patients. Our findings are consistent with those of prior studies in men with CP/CPPS ${ }^{14}$ and women with IC/BPS, ${ }^{8,15}$ but those studies involved detailed, sexspecific examinations. Ours is the first study to propose a simple, sex-neutral examination capable of identifying possible subgroups ("phenotypes") of UCPPS patients. Our extended examination appears to be sensitive enough to capture tenderness both in the pelvis and in extra-pelvic areas. This distinction might be predictive of differences in treatment response, warranting further focused investigation. We emphasize that we found no sex-specific differences between cases and controls.

A secondary finding was that cases had intact pelvic somatosensory innervation, as assessed by genital sensory and reflex testing of the pudendal nerve branches. These findings corroborate an earlier study of men with UCPPS, in which electrophysiological findings did not identify any structural abnormalities of the large fiber pathways of the pudendal nerve. ${ }^{16}$ It appears that large-caliber, myelinated somatic nerve injury is unlikely to be involved in the mechanism underlying UCPPS, and that assessment of pudendal nerve motor and sensory function does not appear to be necessary in most patients with UCPPS. Moreover, if a patient shows evidence of pudendal neuropathy on physical examination, that finding might challenge a diagnosis of UCPPS, since it is a "diagnosis of exclusion."

Despite the presence of identifiable findings, many clinicians believe that physical examination is generally not helpful in evaluating UCPPS ${ }^{17}$ or other chronic pain syndromes, such as chronic fatigue syndrome, ${ }^{18}$ absent pathognomonic findings. However, a physical examination is necessary to rule out identifiable and potentially reversible causes of pain, and it might help to identify patients who will respond to specific therapeutic approaches. For example, when UCPPS is diagnosed, physical findings could be used to direct treatment toward factors that are more likely to contribute to pain. Such treatment might include pelvic floor physical therapy for pelvic floor myalgia or tenderness ${ }^{9,19}$ and systemic pain therapies for more generalized pain beyond the pelvis. 
Our findings raise important questions that merit further research. Do patients' pain symptoms correlate with tenderness on physical examination? Does tenderness predict the natural progression of UCPPS? Should patients with pelvic tenderness be treated differently from patients without such tenderness? A recent study in women with IC/BPS suggested that an extended tender point examination is feasible and tolerable, and can distinguish patient subgroups. ${ }^{15}$ Another study in men found that an extended tender point examination could confirm or rule out CP/CPPS. ${ }^{20}$ While those studies were sex-specific, their results are consistent with our suggestion that musculoskeletal assessment is useful in evaluating UCPPS.

Our findings also demonstrate that an extended physical examination, including assessment of pelvic and extra-pelvic muscular tenderness, can reveal differences between patients with and without UCPPS (as validated by results from both positive and healthy controls). Such differences might help to distinguish subgroups of UCPPS patients; suggest more effective, patient-specific treatment strategies; and improve patient outcomes. Data from the MAPP Research Network have already identified two clinically relevant subgroups: patients reporting "pelvic pain only" and patients reporting "pelvic pain and beyond. ${ }^{21}$ " It remains to be seen if our findings of pelvic floor and extra-pelvic tenderness, as elicited by palpation during the extended examination, are associated with patient reports of pain in the same regions. However, even though the findings on the extended examination are not directly related to particular outcomes at this time, the examination is still clinically relevant, and can be administered and findings documented in the clinic.

We acknowledge certain limitations in our research. Notably, ours was a single-site study with a small participant sample recruited from a tertiary care center. Nonetheless, the limitations associated with a single site are mitigated by the fact that we were conducting a proof of concept study for our approach to physical examination, which is intended for use in most clinical settings. Because the examiners were not blinded to the subjects' status, observer bias could be considered a limitation in this study. Again, determining the feasibility of this examination for clinicians was the objective of this study, and clinicians are not going to be blinded to the status of their patients; physical examination findings are always going to be considered in the context of the medical history and other data. More concerning is the possibility that recruitment from a tertiary care center might not accurately reflect the general population. Thus, future assessment in more representative samples is needed. Finally, we note that the statistically significant differences we found between UCPPS cases and controls are not intended to designate pathognomonic findings for UCPPS, and will require confirmation in larger studies.

\section{Conclusions}

An extended genitourinary examination is an easily administered supplement to the assessment of men and women for UCPPS. Pelvic floor muscle tenderness is more prevalent in UCPPS cases compared to controls. None of our findings were specific to a particular sex, and we saw no evidence of pudendal neuropathy among cases. Further research is needed to determine whether an extended examination leads to better categorization of UCPPS patients into subgroups likely to respond to targeted therapies. 


\section{Supplementary Material}

Refer to Web version on PubMed Central for supplementary material.

\section{Acknowledgments}

Raymond Harris, PhD for critical review of the manuscript.

Grant Funding: NIH 1 U01 DK082325 (MAPP Research Network)

\section{References}

1. Www.mappnetwork.org.

2. Clemens JQ, Mullins C, Kusek JW, et al. The MAPP research network: a novel study of urologic chronic pelvic pain syndromes. BMC Urology. 2014; 14:57. [PubMed: 25085007]

3. Krieger JN, Stephens AJ, Landis JR, et al. Relationship between chronic nonurological associated somatic syndromes and symptom severity in urological chronic pelvic pain syndromes: baseline evaluation of the MAPP study. The Journal of Urology. 2015; 193(4):1254-1262. [PubMed: 25444992]

4. Segura JW, Opitz JL, Greene LF. Prostatosis, prostatitis or pelvic floor tension myalgia? The Journal of Urology. 1979; 122(2):168-169. [PubMed: 459008]

5. Zermann DH, Ishigooka M, Doggweiler R, Schmidt RA. Neurourological insights into the etiology of genitourinary pain in men. The Journal of Urology. 1999; 161(3):903-908. [PubMed: 10022711]

6. Hetrick DC, Ciol MA, Rothman I, Turner JA, Frest M, Berger RE. Musculoskeletal dysfunction in men with chronic pelvic pain syndrome type III: a case-control study. The Journal of urology. 2003; 170(3):828-831. [PubMed: 12913709]

7. Bassaly R, Tidwell N, Bertolino S, Hoyte L, Downes K, Hart S. Myofascial pain and pelvic floor dysfunction in patients with interstitial cystitis. International Urogynecology Journal. 2011; 22(4): 413-418. [PubMed: 20976441]

8. Peters KM, Carrico DJ, Kalinowski SE, Ibrahim IA, Diokno AC. Prevalence of pelvic floor dysfunction in patients with interstitial cystitis. Urology. 2007; 70(1):16-18.

9. FitzGerald MP, Payne CK, Lukacz ES, et al. Randomized multicenter clinical trial of myofascial physical therapy in women with interstitial cystitis/painful bladder syndrome and pelvic floor tenderness. The Journal of Urology. 2012; 187(6):2113-2118. [PubMed: 22503015]

10. Gottsch HP, Yang CC, Berger RE. A pilot study of botulinum toxin A for male chronic pelvic pain syndrome. Scand J Urol Nephrol. 2011; 45(1):72-76. [PubMed: 21062115]

11. Landis JR, Williams DA, Lucia MS, et al. The MAPP research network: design, patient characterization and operations. BMC Urology. 2014; 14:58. [PubMed: 25085119]

12. www.R-project.org. Accessed June 9, 2017.

13. Wolfe F, Smythe HA, Yunus MB, et al. The American College of Rheumatology 1990 Criteria for the Classification of Fibromyalgia. Report of the Multicenter Criteria Committee. Arthritis Rheum. 1990; 33(2):160-172. [PubMed: 2306288]

14. Shoskes DA, Berger R, Elmi A, et al. Muscle tenderness in men with chronic prostatitis/chronic pelvic pain syndrome: the chronic prostatitis cohort study. The Journal of Urology. 2008; 179(2): 556-560. [PubMed: 18082223]

15. Sanses TV, Chelimsky G, McCabe NP, et al. The Pelvis and Beyond: Musculoskeletal Tender Points in Women with Chronic Pelvic Pain. The Clinical Journal of Pain. 2015; 32(8):659-65.

16. Lee JC, Yang CC, Kromm BG, Berger RE. Neurophysiologic testing in chronic pelvic pain syndrome: a pilot study. Urology. 2001; 58(2):246-250. [PubMed: 11489711]

17. Messing E, Pauk D, Schaeffer A, et al. Associations among cystoscopic findings and symptoms and physical examination findings in women enrolled in the Interstitial Cystitis Data Base (ICDB) Study. Urology. 1997; 49(5A Suppl):81-85. [PubMed: 9146006] 
18. Institute of Medicine. Beyond Myalgic Encephalomyelitis/Chronic Fatigue Syndrome: Redefining an Illness. Washington DC: National Academies Press; 2015.

19. Shoskes DA, Nickel JC, Kattan MW. Phenotypically directed multimodal therapy for chronic prostatitis/chronic pelvic pain syndrome: a prospective study using UPOINT. Urology. 2010; 75(6):1249-1253. [PubMed: 20363491]

20. Berger RE, Ciol MA, Rothman I, Turner JA. Pelvic tenderness is not limited to the prostate in chronic prostatitis/chronic pelvic pain syndrome (CPPS) type IIIA and IIIB: comparison of men with and without CP/CPPS. BMC urology. 2007; 7:17. [PubMed: 17908331]

21. Lai HH, Jemielita T, Sutcliffe S, et al. Characterization of Whole Body Pain in Urological Chronic Pelvic Pain Syndrome at Baseline: A MAPP Research Network Study. The Journal of Urology. 2017 Sep; 198(3):622-631. [PubMed: 28373134] 


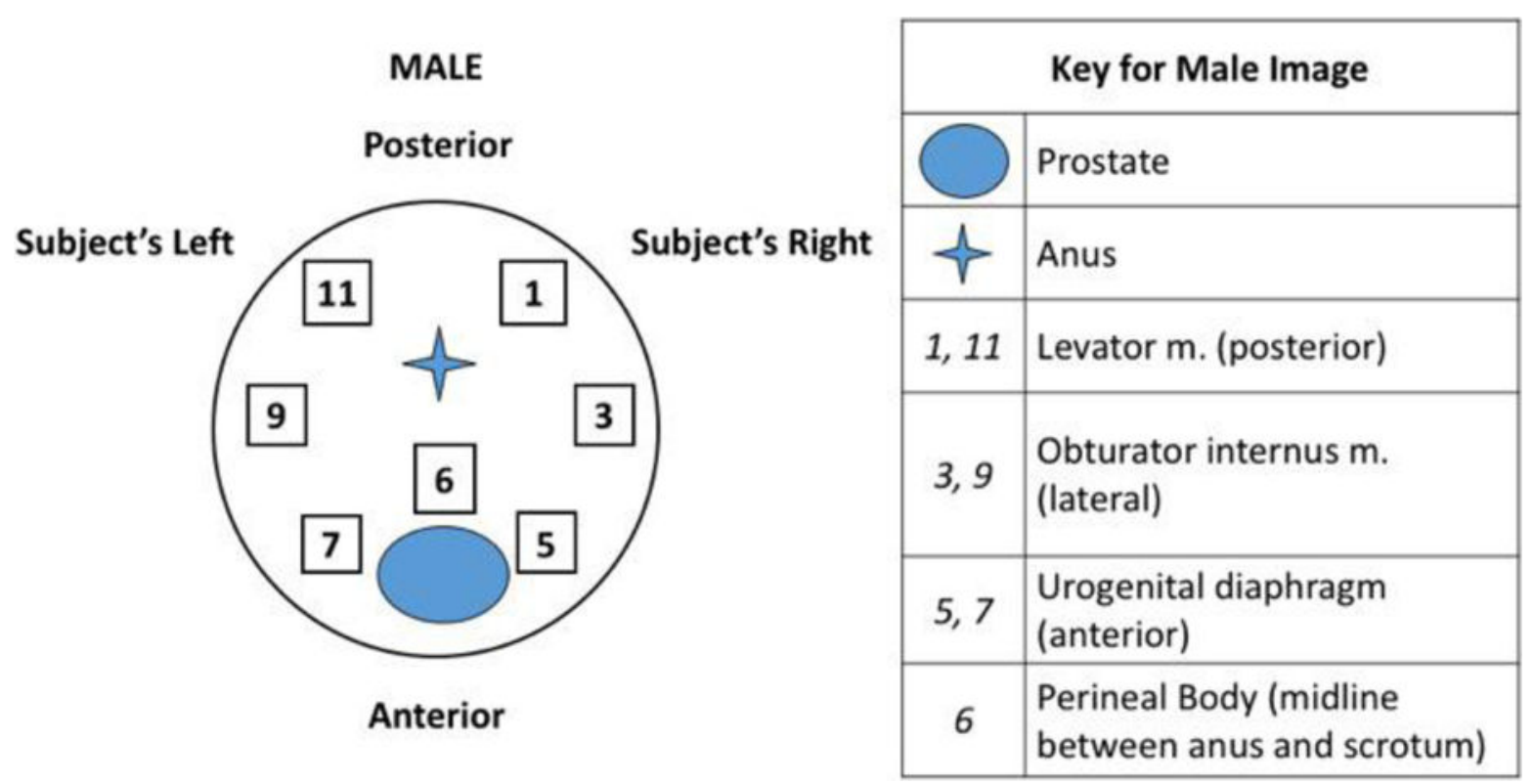

Figure 1.

Diagram for male pelvic muscle examination. Sites of palpation, performed through the rectum, with subject's anterior surface facing down relative to examiner. Numbers correspond to clock-face positions. 
FEMALE

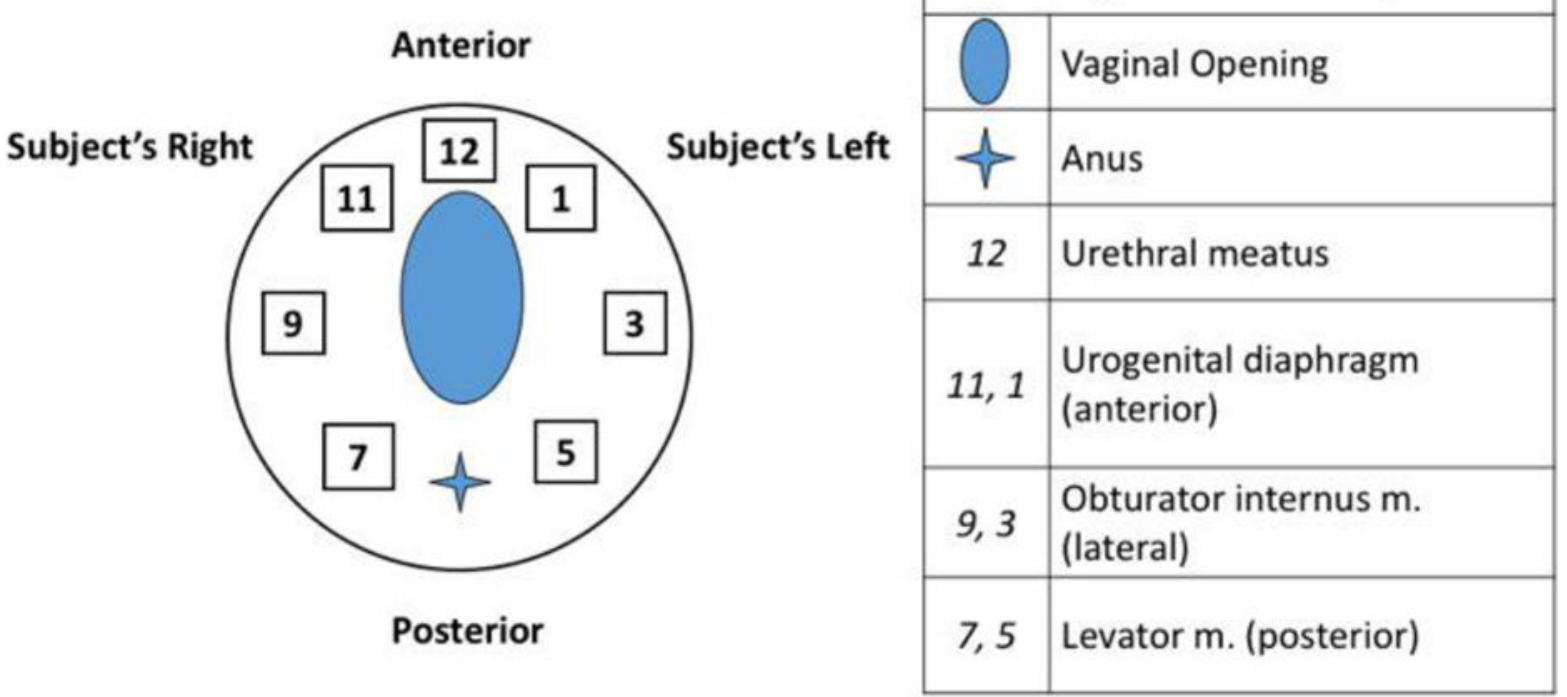

Figure 2.

Diagram for female pelvic muscle examination. Sites of palpation, performed through vagina, with subject's anterior surface facing up relative to examiner. Numbers correspond to clock-face positions. 


\section{Table 1}

Characteristics of Study Participants

\begin{tabular}{|c|c|c|c|}
\hline & $\begin{array}{l}\text { UCPPS Cases } \\
\quad(\mathbf{N}=62)\end{array}$ & $\begin{array}{l}\text { Positive Controls } \\
\quad(\mathbf{N}=\mathbf{4 2})\end{array}$ & $\begin{array}{l}\text { Healthy Controls } \\
\qquad(\mathbf{N}=39)\end{array}$ \\
\hline Age, mean (SD) & $42.7(13.9)$ & $44.5(11.4)$ & $44.2(13.2)$ \\
\hline \multicolumn{4}{|l|}{ Sex, n (\%) } \\
\hline Female & $30(48.4)$ & $15(35.7)$ & $22(56.4)$ \\
\hline Male & $32(51.6)$ & $27(64.3)$ & $17(43.6)$ \\
\hline \multicolumn{4}{|l|}{ Race, n (\%) } \\
\hline White & $44(71.0)$ & $34(81.0)$ & $31(79.5)$ \\
\hline Black & $3(4.8)$ & $5(11.9)$ & $2(5.1)$ \\
\hline Asian & $1(1.6)$ & $1(2.4)$ & $2(5.1)$ \\
\hline Pacific Islander & $0(0.0)$ & $0(0.0)$ & $0(0.0)$ \\
\hline American Indian or Alaska Native & $1(1.6)$ & $1(2.4)$ & $0(0.0)$ \\
\hline Other & $5(8.1)$ & $1(2.4)$ & $1(2.6)$ \\
\hline Did not respond & $8(12.9)$ & $0(0.0)$ & $3(7.7)$ \\
\hline \multicolumn{4}{|l|}{ Ethnicity, n (\%) } \\
\hline No response & $9(14.5)$ & $1(2.4)$ & $3(7.7)$ \\
\hline Hispanic or Latino & $3(4.8)$ & $1(2.4)$ & $3(7.7)$ \\
\hline Not Hispanic or Latino & $50(80.6)$ & $40(95.2)$ & $33(84.6)$ \\
\hline \multicolumn{4}{|l|}{ Employment, n (\%) } \\
\hline Employed & $43(69.4)$ & $14(33.3)$ & $26(66.7)$ \\
\hline Unemployed & $6(9.7)$ & $11(26.2)$ & $6(15.4)$ \\
\hline Retired & $3(4.8)$ & $3(7.1)$ & $5(12.8)$ \\
\hline Homemaker & $3(4.8)$ & $1(2.4)$ & $2(5.1)$ \\
\hline Disabled & $7(11.3)$ & $13(31.0)$ & $0(0.0)$ \\
\hline Married or with partner, n (\%) & $40(64.5)$ & $15(35.7)$ & $15(38.5)$ \\
\hline Less than 2 years duration of pelvic pain symptoms, $\mathrm{n}(\%)$ & $36(58.1)$ & $0(0.0)$ & $0(0.0)$ \\
\hline BMI, mean (SD) & $26.7(6.5)$ & $27.4(8.8)$ & $26.1(4.4)$ \\
\hline \multicolumn{4}{|l|}{ BMI (CDCP categorization), n (\%) } \\
\hline Normal BMI & $25(41.0)$ & $16(38.1)$ & $17(44.7)$ \\
\hline Overweight & $25(41.0)$ & $14(33.3)$ & $15(39.5)$ \\
\hline Obese & $11(18.0)$ & $12(28.6)$ & $6(15.8)$ \\
\hline
\end{tabular}

Abbreviation: $\mathrm{BMI}=$ body mass index $\mathrm{CDCP}=$ Centers for Disease Control and Prevention . 


\section{Table 2}

Physical Examination Findings in Cases of Urological Chronic Pelvic Pain Syndrome and Age- and Sexmatched Controls

\begin{tabular}{|c|c|c|c|}
\hline & $\begin{array}{l}\text { UCPPS Cases } \\
\quad(\mathbf{N}=62)\end{array}$ & $\begin{array}{l}\text { Positive Controls } \\
\quad(\mathrm{N}=\mathbf{4 2})\end{array}$ & $\begin{array}{l}\text { Healthy Controls } \\
\quad(\mathbf{N}=39)\end{array}$ \\
\hline \multicolumn{4}{|l|}{ EP Examinations, $\mathrm{n}(\%)^{\#}$} \\
\hline Abdominal exam abnormal & $13(22.4)$ & $6(14.3)$ & $0(0.0) *$ \\
\hline External genitalia abnormal & $7(11.5)$ & $2(4.8)$ & $1(2.6)$ \\
\hline Rectal/bimanual abnormal & $9(15.3)$ & $5(12.8)$ & $1(2.7)$ \\
\hline Pelvic floor tenderness & $33(55.0)$ & $6(14.6)^{*}$ & $4(10.5)^{*}$ \\
\hline Suprapubic tenderness (M) & $4(12.5)$ & $3(11.1)$ & $0(0.0)$ \\
\hline Prostate tenderness (M) & $7(21.9)$ & $0(0.0)$ & $0(0.0)$ \\
\hline Penis circumcised (M) & $24(75.0)$ & $21(77.8)$ & $13(76.5)$ \\
\hline Varicocele present (M) & $1(3.1)$ & $1(3.7)$ & $0(0.0)$ \\
\hline Hydrocele present (M) & $0(0.0)$ & $0(0.0)$ & $0(0.0)$ \\
\hline Mass of testis/epididymis (M) & $0(0.0)$ & $0(0.0)$ & $2(11.8)$ \\
\hline Uterus present $(\mathrm{F})$ & $23(76.7)$ & $13(92.9)$ & $21(95.5)$ \\
\hline Prolapse present, no vaginal points beyond hymen (F) & $4(16.0)$ & $0(0.0)$ & $1(5.9)$ \\
\hline Prolapse present, one or more vaginal points beyond hymen $(\mathrm{F})$ & $0(0.0)$ & $0(0.0)$ & $0(0.0)$ \\
\hline \multicolumn{4}{|l|}{$\begin{array}{l}\text { Extended Examination } \\
\text { Tenderness Present, n (\%) }\end{array}$} \\
\hline Rectus muscles & $7(12.5)$ & $5(11.9)$ & $0(0.0)$ \\
\hline Suprapubic region & $13(23.2)$ & $1(2.4) *$ & $0(0.0)$ * \\
\hline Symphysis pubis & $10(17.9)$ & $2(4.8)$ & $0(0.0) *$ \\
\hline Paravertebral muscles & $7(11.7)$ & $3(7.1)$ & $0(0.0)$ \\
\hline Posterior superior iliac spine & $10(16.9)$ & $3(7.3)$ & $0(0.0) *$ \\
\hline Coccyx & $7(13.5)$ & $3(7.3)$ & $0(0.0)$ \\
\hline Bimanual exam (any urge or tenderness) & $24(92.3)$ & $8(88.9)$ & $1(7.7)^{*}$ \\
\hline Perineal body & $0(0.0)$ & $2(11.1)$ & $0(0.0)$ \\
\hline \multicolumn{4}{|l|}{ Levator muscles } \\
\hline Right & $23(40.4)$ & $5(12.2)^{*}$ & $1(2.7) *$ \\
\hline Left & $19(33.3)$ & $5(12.2) *$ & $1(2.7) *$ \\
\hline Any $\xi$ & $26(45.6)$ & $5(12.2) *$ & $2(5.4)^{*}$ \\
\hline \multicolumn{4}{|l|}{ Obturator muscles } \\
\hline Right & $21(36.8)$ & $4(9.8)^{*}$ & $0(0.0) *$ \\
\hline Left & $19(33.3)$ & $3(7.3) *$ & $0(0.0)$ * \\
\hline Any $\mathcal{\xi}$ & $23(40.4)$ & $4(9.8) *$ & $0(0.0)$ * \\
\hline Urogenital diaphragm & & & \\
\hline
\end{tabular}

Urology. Author manuscript; available in PMC 2019 June 01. 


\begin{tabular}{lccc}
\hline & $\begin{array}{c}\text { UCPPS Cases } \\
(\mathbf{N}=\mathbf{6 2})\end{array}$ & $\begin{array}{c}\text { Positive Controls } \\
(\mathbf{N}=\mathbf{4 2})\end{array}$ & $\begin{array}{c}\text { Healthy Controls } \\
(\mathbf{N}=\mathbf{3 9})\end{array}$ \\
\hline Right & $21(36.8)$ & $5(12.2)$ & $0(0.0)^{*}$ \\
\hline Left & $19(33.3)$ & $3(7.3)^{*}$ & $0(0.0)$ \\
\hline Any $\xi$ & $23(40.4)$ & $5(12.2)^{*}$ & $0(0.0)^{*}$ \\
\hline
\end{tabular}

Abbreviation: EP = Epidemiology and Phenotyping study

\# EP examinations: "abnormal" not defined in the protocol and left to the investigator's discretion; (M) and (F) are designations for examinations performed on males or females only.

Indicates Pearson's chi-squared test p-value $<0.05$, compared to cases.

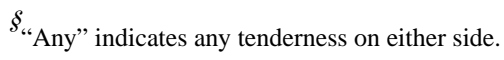

